\title{
Fabrication of Carbon Nanotubes and Investigating the Characteristics
}

\author{
T. Alex Benhur Roche, B.E., M.E. \\ Dept. of Electronics and Telecommunication Eng. \\ Sathyabama University \\ Chennai India
}

\author{
S. Maflin Shaby, M.E., Ph.D. \\ Assistant Professor, Dept. of ETCE \\ Sathyabama University \\ Chennai India
}

\begin{abstract}
Carbon nanotubes (CNT) are the fantastic nano-material which exhibits a very low thermal expansion and high current capability. It has a wide variety of methods for synthesis. One of the most reliable and best suited is Arc Discharge method. Multi walled CNT (MWCNT) are synthesized in an experimental setup. Soot deposited is in mixture form of nanoparticles and hence it is purified and MWCNT are separated and collected. XRD analysis and TEM images are taken for CNT. MWCNT are coated on the silica substrate through DIP coating. Dispersion of CNT is handled and the resistivity and morphology of cnt dip coated are studied. The change in resistivity is determined by the graph plotted through Four Point Probe method. Investigation concludes that the MWCNT developed is suitable for contacts in transistors and miniature sensors for fabrication due to its low resistivity.
\end{abstract}

\section{General Terms}

Carbon Nanotubes, synthesis, purification and characterization

\section{Keywords}

Carbon nanotubes, Arc discharge method, synthesis, purification, characterization - XRD, SEM, TEM.

\section{INTRODUCTION}

Nowadays our world is full of innovation and intelligence. To suit the expectation nanotechnology is a mandatory. Substance at mass behaves different in nanoscale. There is a wide change in characteristics of materials. Nanoscale development is a Most innovative and efficient field, carbon nanotubes are the best option for contacts and nanoscale fabrication.

CNTs have been a subject of fascination for many scientists and engineers. They have received considerable interest in research due to their unique atomic structure/dimensions and exceptional properties.

Interconnects made by copper, aluminum at nanoscale fabrication doesn't have the unique properties of cnt such as low thermal expansion [1] and high current density [2] enables it to suitable for such precise capabilities. CNT due to its structure and conductivity properties it has become an effective research and development area.

Nanotubes have a cylindrical structure and it can be synthesized through different methods. Synthesis of CNT is done basically through Arc discharge method. Arc discharge method is Easy \& best suited for laboratory preparation which yields High purity Multi Walled Carbon Nanotubes
(MWCNT). It is comparatively cheaper than Laser method and easier than Chemical method.

In this arc discharge method the carbon soot is produced which has nanotubes, nano particles etc.., the carbon soot cannot be used as such so it is purified. Purification yield pure $\mathrm{CNT}$ and efficient nanotubes. This process includes grinding, soaking, filtering, drying and cooling.

Characterization is the conformation of CNT and justification of nanotubes structure, size and morphology. The X-Ray diffraction method is done to prove the presence of Graphite. The Travelling Electron Microscope (TEM) displays the size of the nanotubes.

This paper exhibits the best, easy and well suited methodology for multi walled CNT synthesis, purification and characterization. Results and discussion conforms the presence of multi walled carbon nanotubes to a length of $100 \mathrm{~nm}$ and a diameter of $10 \mathrm{~nm}$ are produced. Since CNT is a well suited for high conductive in nature and low thermal expansion in further research dispersion and conductivity may be possible.

\section{SYNTHESIS}

There are various types suited for CNT preparation. Laser method, chemical method, arc discharge method and plasma torch method.

Since arc discharge method [3] is well suited for multi walled $\mathrm{CNT}$ it is taken into consideration. Also it yields more CNT and comparatively more economy.

Arc discharge method parameters are listed. Basically this method yield a high purified multi walled CNT.

$$
\begin{array}{lll}
\text { - } & \text { Material used } & : \text { Graphite rod } \\
\text { - } & \text { Rod Size (Anode) } & : 30 \mathrm{~cm} \text { length \& } 11 \mathrm{~mm} \text { diameter } \\
\text { - } & \text { Disc Size (Cathode) }: 50 \text { x } 10 \mathrm{~mm} \text { disc } \\
\text { - } & \text { Atmosphere } & : \text { Nitrogen } \\
\text { - } & \text { Current } & : 150 \text { Amperes } \\
\text { - } & \text { Voltage } & : 30 \mathrm{~V} \mathrm{DC} \\
\text { - } & \text { Speed } & : 10 \mathrm{rpm}
\end{array}
$$

The experimental setup includes nitrogen filled chamber with water cooling system, servo motors and DC Power supply. Fig 1 illustrates the experimental setup. 


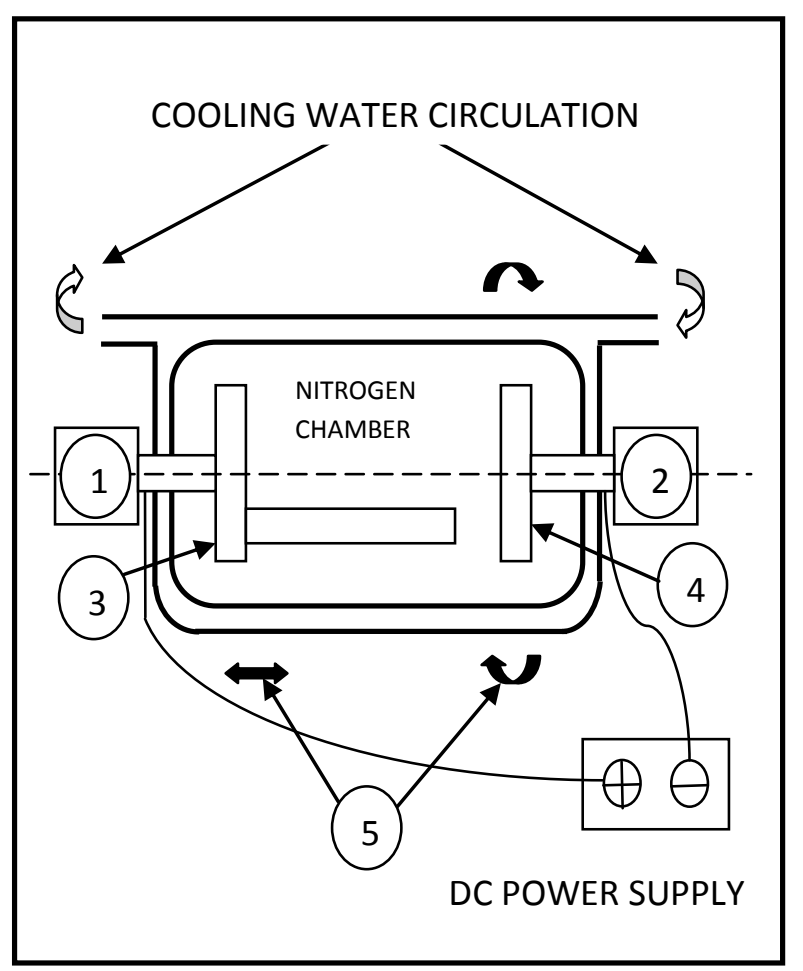

Fig 1: Schematic of Arc Discharge Method Legends:

$$
\begin{aligned}
& \text { 1-Servo Motor } \\
& 2 \text { - DC Motor (10 RPM) } \\
& \text { 3- Graphite Rod as Anode } \\
& \text { 4- Graphite Disc as Cathode } \\
& \text { - Direction of Rotation }
\end{aligned}
$$

Initially the chamber was vacuumized to $600 \mathrm{~mm}$ of $\mathrm{Hg}$ (Mercury) pressure (0.799 bar). Nitrogen was purged to maintain $1 \mathrm{~kg} / \mathrm{cm}^{2}$ (0.980 bar). Graphite rod was drilled with $4 \mathrm{~mm}$ diameter hole and the hole was filled with Nickel (Ni) \& graphite powder which acted as a catalyst in a ratio of 1:1.

Nickel as a catalyst [4] \& [5] is corrosive resistant, heat resistant and controlled expansion alloy. The Melting point and boiling point is comparatively low when compared with other catalyst such as Iron (Fe) and Cobalt (Co). The cost is also less when compared with cobalt alloy.

A DC Power supply is connected to both electrode and disc. Electrode act as Anode and Rotating graphite disc at 10 RPM was used as cathode.

When the specified voltage and current is applied and the gap between anode and cathode is maintained to $1 \mathrm{~mm}$, arc generated between two electrodes (Graphite). Cooling water was circulated to remove the heat.

During discharge carbon atoms sublimate from anode and gets deposited on cathode. A carbonaceous deposit rich in MWCNT (Multi Walled Carbon Nanotubes) grow up.

A servo motor moves the anode forward when the anode gets consumed, in order to maintain the electrode gap constant. Soot deposited on the graphite (cathode) was continuously removed using a thin blade.
By using this thin blade the cathode is always maintained free of deposited material which is constantly removed from the high temperature reaction region.

The collected carbon soot is cooled at room temperature.

\section{PURIFICATION}

Carbon soot contains nanotubes, fibers, particles etc.., this soot cannot be used as such hence it has to be purified. Purification undergoes basically grinding, soaking, filtering, drying, cooling, treating, filtering and heating. Purification steps are represented in a block diagram. Fig 2 illustrates the block diagram of purification.

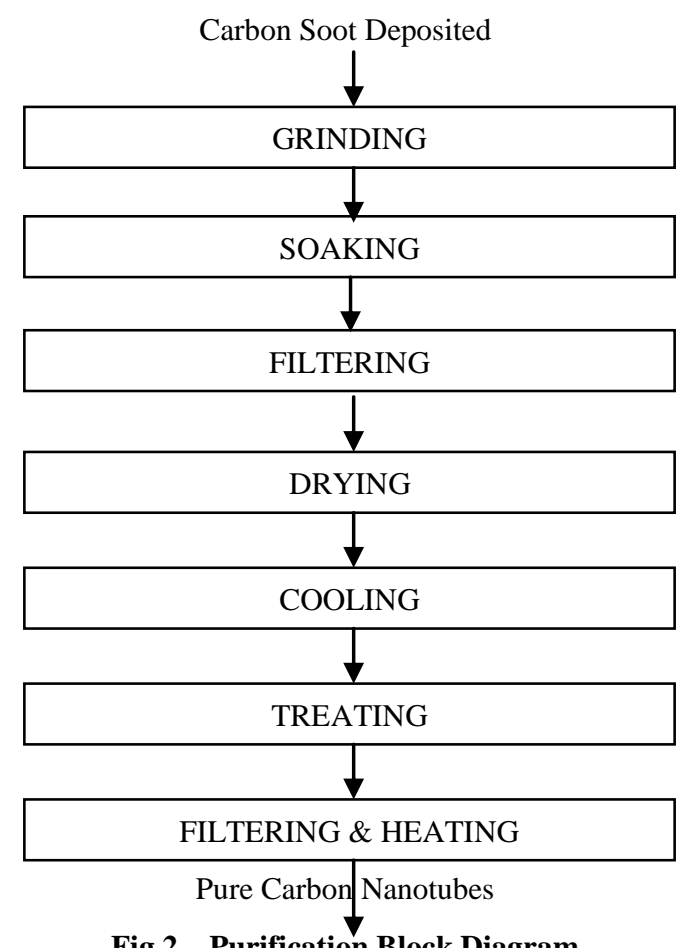

Fig 2 - Purification Block Diagram

\subsection{Grinding}

Pestle and Mortar is used for grinding the carbon soot. Due to discharge the carbon nanotubes are bonded together and to get the carbon nanotubes in powder it is grinded to a fine powder.

\subsection{Soaking}

De-ionized water is used for soaking. This helps in removing suspended particles in the grinded soot. The soaking is done for one hour.

\subsection{Filtering}

High quality Ashless filter papers of grade 42 used to filter the soot. The diameter is $125 \mathrm{~mm}$ circle filter paper and Cat no 1442125 which is well suited.

\subsection{Drying and Cooling}

The filtered soot is kept in an oven at $120^{\circ} \mathrm{C}$. The time the soot is kept is one hour. Then the mass is cooled at room temperature.

\subsection{Treating, Filtering and Heating}

The soot is treated with toluene solvent for 3hours at a ratio of 1:3. The CNT are again Filtered and Heated in an oven at $85^{\circ} \mathrm{C}$ for 1 hour. Again the treated soot is heated in air tubular furnace at $480^{\circ} \mathrm{C}$ for 3 hours followed by air cooling. 


\section{DISPERSION}

Dispersion [6] is a method of splitting combined carbon nanotubes. Multi walled Carbon nanotubes (MWCNTs) obtained are compressive together and for coating it has to be individualized and hence they are taken and treated with sulfuric acid $\left(\mathrm{H}_{2} \mathrm{SO}_{4}\right)$ at $30 \%$ wt concentration in water for 15 min at $60^{\circ} \mathrm{C}$ in an oven. The treated cnt is washed in distilled water for 3 times and filtered in the Ashless filter paper. The filtered cnt is dried in oven for $10 \mathrm{~min}$

$75 \mathrm{mg}$ of cnt is dispersed in $50 \mathrm{ml}$ of acetone and sonically treated for 8 hours. Similarly $50 \mathrm{mg}$ of cnt is dispersed in $100 \mathrm{ml}$ of acetone and sonically treated for 8 hours. CNT dispersed solution is obtained and made ready for coating and it is shown in fig 4.

Parameters for CNT Dispersion:

- $\quad$ MWCNT $125 \mathrm{mg}$

- $\mathrm{H}_{2} \mathrm{SO}_{4}$

$$
\begin{array}{ll}
- & 30 \mathrm{ml} \\
- & 200 \mathrm{ml}
\end{array}
$$

- $\quad$ Acetone solvent $\left(\mathrm{C}_{3} \mathrm{H}_{6} \mathrm{O}\right)$

- Oven

- $\quad$ Distilled water

$-\quad 500 \mathrm{ml}$

A $15 \mathrm{ml}$ of dispersed MWCNT in acetone solvent at different percentage is collected and stored in an air tight bottle. Fig 5 shows the different percentage dispersed cnt.

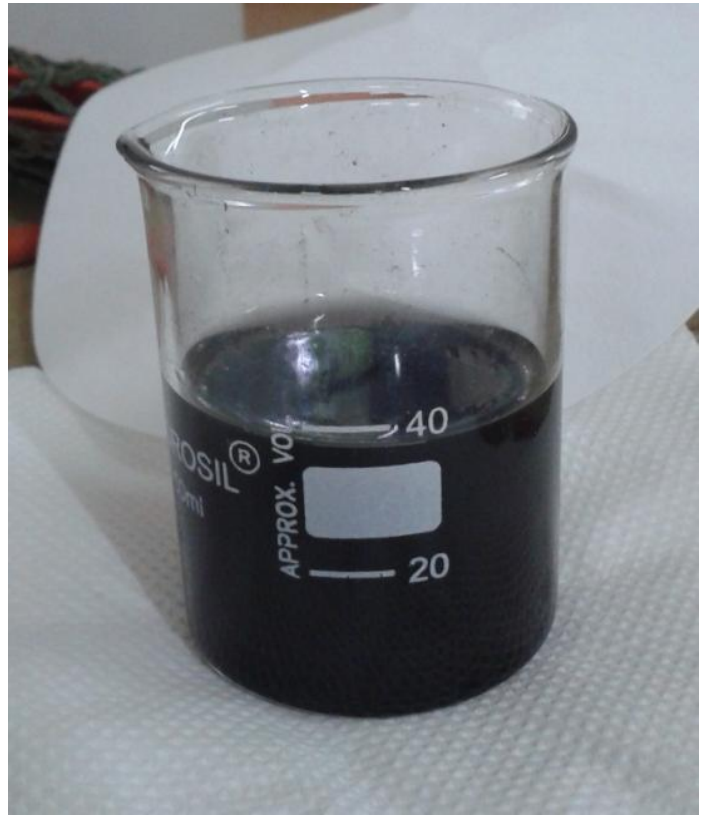

Fig 4 - CNT dispersed in Acetone

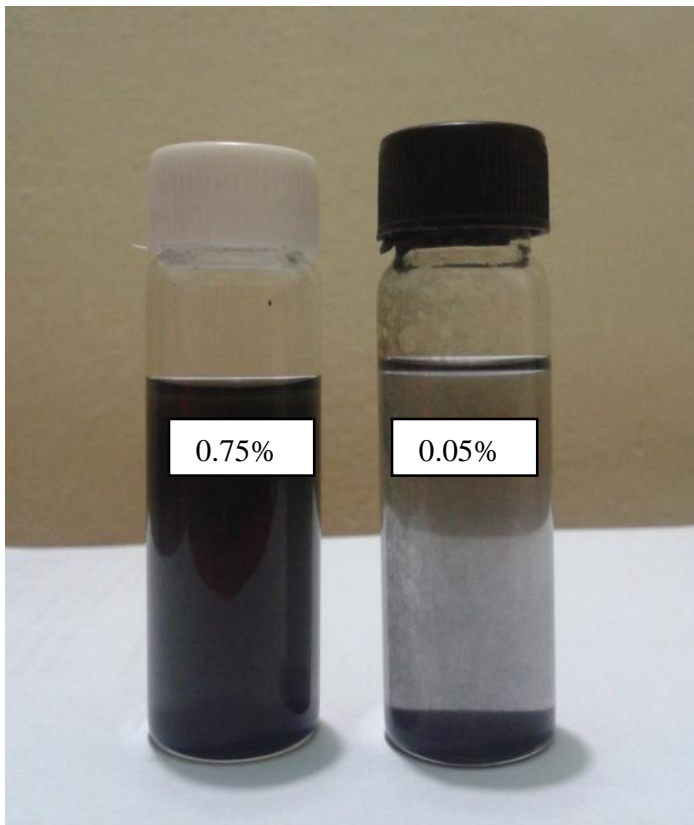

Fig $5-0.75 \%$ \& $0.05 \%$ dispersed MWCNT

\section{COATING}

There are many coating techniques possible such as spin coating, DIP coating [6], Ion Implantation etc.., DIP coating is an easy and reliable which involves solvent dispersion.

Parameters for DIP coating:

- $\quad$ Ethanol absolute $-30 \mathrm{ml}$

- $\quad$ Distilled water

$-1000 \mathrm{ml}$

- Substrate - Fused Quartz / Silica

- $\quad$ DIP coating machine \& Oven

The substrate is pre-cleaned through ethanol absolute. It is sonically treated for 1 hour and washed with distilled water 3 times. Now the substrate is dipped in the dispersion liquid for $5 \mathrm{~min}$ for first cycle and then dried in oven at $50^{\circ} \mathrm{C}$.

5 samples are taken and after first cycle one sample is removed from oven. For second cycle again the four samples are dip coated and dried in oven. After drying again one sample is removed.

Similarly from one cycle to five cycle dip coated sample are attained. Through this dip coating cycles the cnt coating thickness is varied from $100 \mathrm{~nm}$ to $250 \mathrm{~nm}$. Fig 6.a and Fig 6.b shows the DIP coating of carbon nanotubes in silica substrate. SEM image shows the CNT is coated on silica and it is connected together to form the electrical conductivity. 


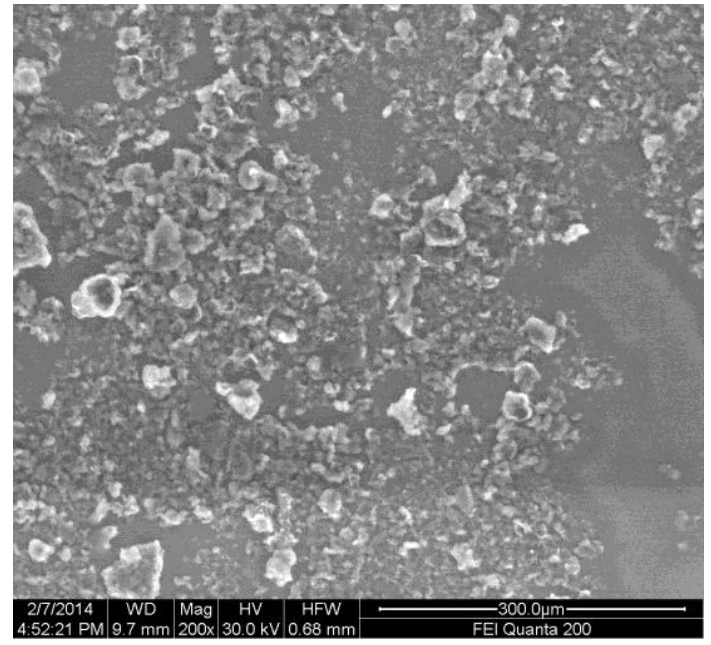

Fig 6.a. 500 $\mu \mathrm{m}$ SEM image of Dip coated CNT

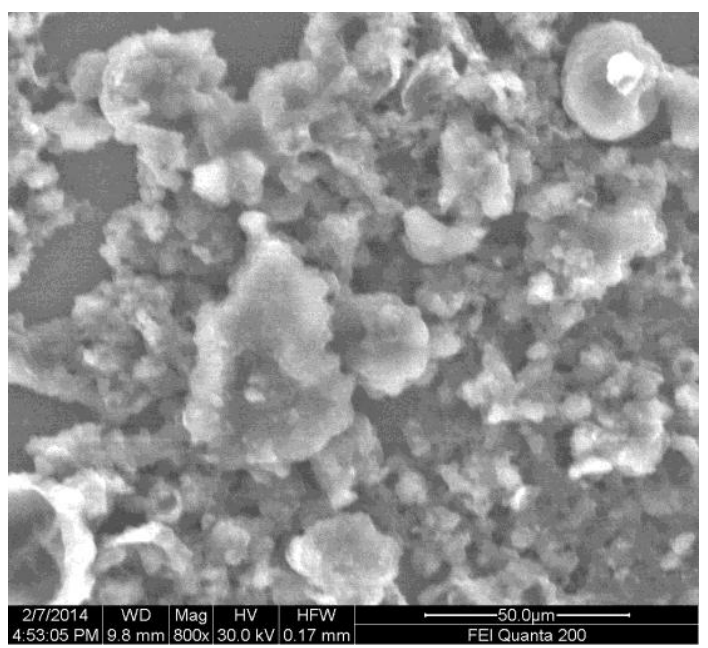

Fig 6.b. 50 $\mathrm{\mu m}$ SEM image of Dip coated CNT

\section{CHARACTERISATION}

Carbon nanotubes are to be characterized by its size, structure, resistivity etc.., Characterization of CNT involves mainly

- $\quad \mathrm{XRD}$ analysis

- $\quad$ TEM image morphology

- Four point Probe Method

\subsection{XRD Analysis}

X-ray crystallography tool or XRD analysis [7] is used to determine atomic and molecular structure. The atoms cause the beam of X-rays to diffract in many directions. Depending on angles and intensities of diffracted beams the crystallographer produces a 3D picture of density of electrons.

The electron density shows mean positions, chemical bonds and disorder of atoms in the crystal. The first decade used this method to determine the atom size, lengths, chemical bonds types; atomic-scale in various minerals, alloys etc.., It is still a great method to characterize atomic structure of new materials. It is also suited to the properties of material.

The measurement is made through a crystal mounted to goniometer and precisely rotated to bombard with X-rays which produces the diffraction pattern or reflections. Two dimensional images are converted to $3 \mathrm{D}$ using Fourier transforms for the samples.

The diffracted pattern are plotted to identify the availability of carbon nanotubes. Fig 7 illustrates the XRD analysis chart. From XRD it was observed that produced CNTs are crystalline in nature graphitic (002) peak corresponding to the pure graphitic reflection

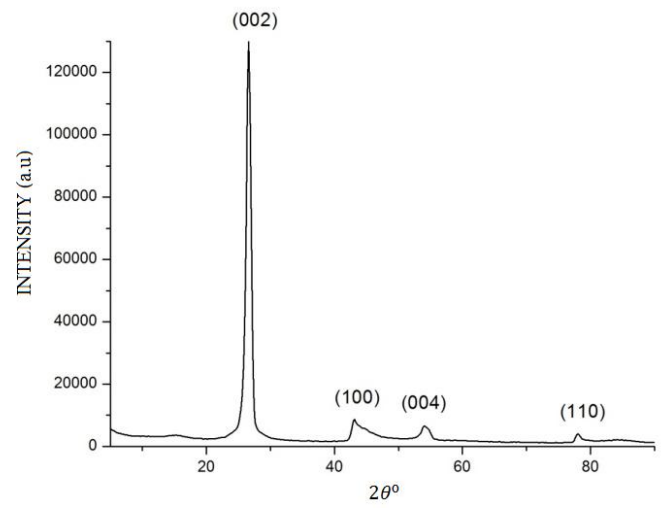

Fig 7 - X-Ray Diffraction pattern for MWCNT

\subsection{Transmission Electron Microscopy (TEM) Analysis:}

It is a technique where a beam of electrons are transmitted through the specimen as it passes through. The image formed due to interaction of electrons are magnified and focused to an imaging device like fluorescent screen on a layer of the film or detected in CCD camera.

Its capability of imaging is significantly higher than light microscopes, owing to very less de Broglie wavelength of electrons. This helps the instrument user to examine fine details like a single column of atoms.

It can measure thousands of times smaller than the smallest resolvable object in light microscope. It forms a major analysis method of range in scientific fields for both physical and biological sciences.

The TEM [8] image of the Carbon nanotubes at two different scales is illustrated in Fig 8 and Fig 9. While analyzing the nanometer scale of the MWCNT it indicates the Carbon nanotubes are $99 \%$ pure.

The Fig 8 shows morphology analysis shows that the MWCNT are properly synthesized. The nanotubes length are measured and found to be approx $100 \mathrm{~nm}$.

Since CNT is a conductive in nature it can be used for contacts in fabrication. Further scaled image of the CNT is also done and the diameter of the nanotubes is measured and it is approx $10 \mathrm{~nm}$. Fig 9 shows the $50 \mathrm{~nm}$ scaled image. 


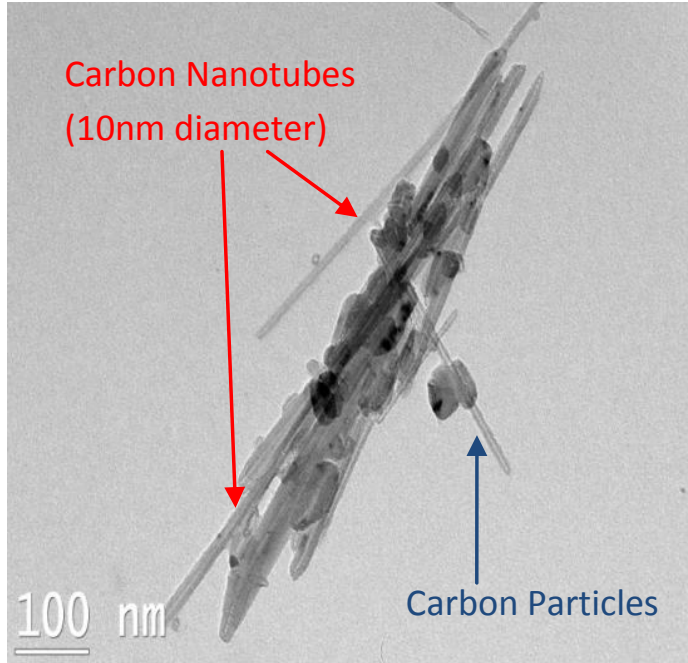

Fig 8 - 100nm scale TEM image of MWCNT

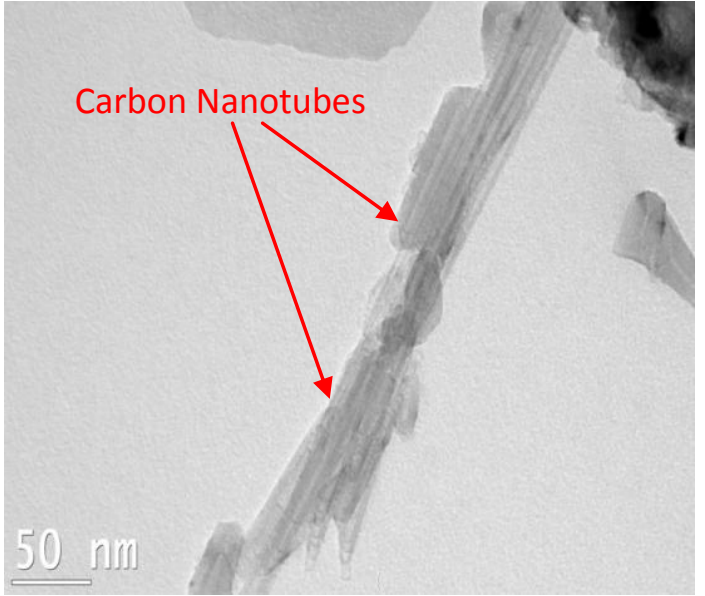

Fig 9 - 50nm scale TEM image of MWCNT

\subsection{Electrical Resistivity of CNT}

Electrical resistance of $\mathrm{cnt}$ is a factor to analyze the conductivity. Depending on the resistance of the CNT it can be classified. To calculate the resistivity four point probe method [9] \& [10] is used the purpose of it is to measure the resistivity of any semiconductor. It measures either bulk or thin film specimen, which has different expression. It has four equally spaced tungsten metal tips with finite radius. Each tip is supported by springs to minimize sample damage.

A current source which has high impedance is used to supply current through the outer two probes. A voltmeter measures the voltage across the inner two probes to find the sample resistivity. For a very thin layer $\mathrm{s}<<1 \mathrm{~mm}$ we have current rings instead of spheres. Deriving and solving we get the equation,

Resistivity, $\mathrm{R}=[\pi / \ln (2) * \mathrm{t} *(\mathrm{~V} / \mathrm{I})]$

Where,

$$
\begin{aligned}
& t=\text { thickness of the coating }(t=100 \mathrm{~nm}) \\
& V=\text { Voltage of the layer coated } \\
& I=\text { Current of the layer coated }
\end{aligned}
$$

Fig 10 shows the I-V characteristics. On solving, the resistivity of the cnt is measured as $11 \mathrm{~m}$ ohms, which represent it is highly conductive. The resistivity when compared with a reference papers [11] \& [12] the cnt got is having low resistivity. Hence it is very well suited for high conductivity sensors or interconnects.

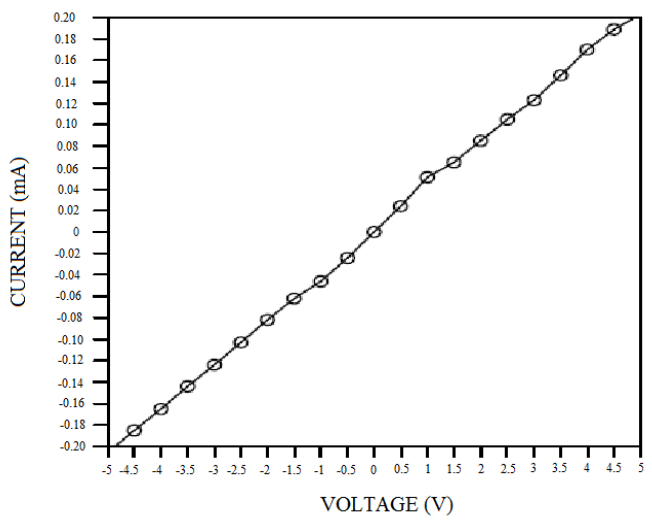

Fig 10 - I-V curve for Electrical Resistivity

\section{RESULTS}

Synthesis of multi walled carbon nanotubes through Arc Discharge method give a promising result and are so reliable. The results show that the MWCNT are synthesized properly and the carbon nanotubes are having a length of $100 \mathrm{~nm}$ and diameter of $10 \mathrm{~nm}$. Resistivity is found as $11 \mathrm{~m}$ ohms and can be used as contacts and sensors used in hydrogen chamber.

\section{DISCUSSION}

Since carbon nanotubes having a cylindrical structure it can be used for composite mixture. A Future approach with composites will be analyzed in further research Tran's conductance of cnt will also be concentrated in further research.

\section{REFERENCES}

[1] H. Jiang, B. Liu, Y. Huang, K. C. Hwang. July 2004. Thermal expansion of single wall carbon nanotubes. Journal Eng. Mater., Technology

[2] P. G. Collins, M. Hersam, M. Arnold, R. Martel, and P. Avouris. April 2001. Current saturation and electrical breakdown in multiwalled carbon nanotubes. Physics Revolution Letter.

[3] Yu-Hsiang Wang, Sheng-Cheng Chiu, Kuo-Min Lin, Yuan-Yao Li. 2004. Formation of carbon nanotubes from polyvinyl alcohol using arc-discharge method. Elservier Limited.

[4] Joydip Sengupta, Chacko Jacob. 2009. The effect of Fe and $\mathrm{Ni}$ catalysts on the growth of multiwalled carbon nanotubes using chemical vapour deposition. Springer Science Business Media

[5] Muhammad Musaddique Ali, Rafique, Javed Iqbal. 2011. Production of Carbon Nanotubes by Different RoutesA Review. Journal of Encapsulation \& Adsorption Sciences.

[6] Zuo-Jia Wang a, Dong-Jun Kwon a, et. Al.. 2012. Surface control and cryogenic durability of transparent CNT coatings on dip-coated glass substrates. Elsevier Limited.

[7] Christophe Pirlot, I. Willems et. Al., 2002. Preparation and Characterisation of Carbon Nanotube / 
Polyacrylonitrile Composite. Advanced Engineering Materials.

[8] K. Safarova, A. Dvorak, et. Al., 2007. Usage of AFM, SEM and TEM for the research of carbon Nanotubes", Formatex.

[9] Wayne Lewis, Chris Brown, and Wilhelmus J. Geerts September 2003. A laboratory on the four-point probe technique.

[10] James Chan and Paul Friedberg. 2002. Four - Point Probe Manual”, Microfabrication Technology.
[11] Teng Wang, Si Chen, Di Jiang, et. Al., March 2012. "Through-Silicon Vias Filled With Densified and Transferred Carbon Nanotube Forests", IEEE Electron Device Letters.

[12] T.Wang, K. Jeppson, L. Ye, and J. Liu. August 2011. Carbon-nanotube through-silicon via interconnects for three-dimensional integration. WILEY-VCH Verlag $\mathrm{GmbH} \&$ Co. K. GaA, Weinheim. 